receiving nebulised pentamidine prophylaxis; ${ }^{45}$ all five HIV positive patients with disseminated extrapulmonary granulomatous pneumocystis infection were receiving both zidovudine and nebulised pentamidine. Possible mechanisms to explain the granulomatous response include an improvement in host defences due to treatment with zidovudine ${ }^{5}$ and a local toxic reaction to nebulised pentamidine; pentamidine isethionate dissolved in water for nebulisation is both acidic and hypo-osmolar. ${ }^{9}$ The pallisade of histiocytes presumably walls off the pneumocystis organisms, rendering them inaccessible during bronchoalveolar lavage and preventing nebulised pentamidine from having a therapeutic effect.

In conclusion, this case shows that a positive gallium-67 citrate scan in a patient with pulmonary Kaposi's sarcoma should prompt a search for an alternative infective or inflammatory pathological process and that pneumocystis infection may be associated with a granulomatous response, which may possibly be induced by zidovudine or nebulised pentamidine.

We thank Jane Healing for typing the manuscript.

1 Suster B, Ackerman M, Orenstein M, Wax M. Pulmonary manifestations of AIDS: review of 106 episodes. Radiology 1986;161:87-93.

2 Ognibene FP, Steis RG, Macher AM, et al. Kaposi's sarcoma causing pulmonary infiltrates and respiratory failure in the acquired immunodeficiency syndrome. Ann Intern Med 1985;102:471-5.

3 Miller RF. Nuclear medicine and AIDS. Eur J Nucl Med 1990;16:103-18.

4 Blumenfeld W, Basgoz N, Owen WF, Schmidt DM. Granulomatous pulmonary lesions in patients with the acquired immunodeficiency syndrome (AIDS) and Pneumocystis carinii infection. Ann Intern Med 1988; 109:505-7.

5 Klein JS, Warnock M, Webb WR, Gamsu G. Cavitating and non-cavitating granulomas in AIDS patients with pneumocystis pneumonitis. AJR 1989;152:753-4.

6 Radin DR, Baker EL, Klatt EC, et al. Visceral and nodal calcification in patients with AIDS-related Pneumocystis carinii infection. AJR 1990;150:27-31.

7 LeGolvan DP, Heidelberger KP. Disseminated granulomatous Pneumocystis carinii pneumonia. Arch Pathol 1973;95:344-8.

8 Cruickshank B. Pulmonary granulomatous pneumocystosis following renal transplantation. Am J Clin Pathol 1975;63:384-90.

9 Miller RF, Semple SJG. Bronchial bleeding from nebulised pentamidine. Lancet 1988;ii:1488.
Department of

Pulmonary Medicine, Roosevelt Hospital (a University Hospital of Columbia University College of Physicians and Surgeons), New York, USA R Minutoli E Eden

C Brachfeld

Address for reprint requests: Dr Edward Eden,

Pulmonary Division,

Roosevelt Hospital, 428

W59th Street, New York,

NY 10019, USA.

Accepted 4 April 1990

\section{Bronchoalveolar lavage via a modified stomach tube in intubated patients with the acquired immuno- deficiency syndrome and diffuse pneumonia}

\author{
Ronald Minutoli, Edward Eden, Claude \\ Brachfeld
}

\begin{abstract}
A simple non-bronchoscopic bronchoalveolar lavage method was used in $\mathbf{3 0}$ patients with the acquired immunodeficiency syndrome undergoing assisted ventilation for respiratory failure. A modified Argyle Levin stomach tube was passed via the endotracheal tube and lavage performed. The lavage was well tolerated and performed quickly and easily, required little training, and had a high degree of sensitivity $(73 \%-a$ diagnosis in 22 of the 30 cases).
\end{abstract}

Pneumonia is a common complication in the acquired immunodeficiency syndrome (AIDS), ${ }^{1-3}$ and is associated with a high morbidity and mortality. Early diagnosis and aggressive treatment improve the outcome. Pneumocystis carinii is the most common pathogen diagnosed, but the differential diagnosis of diffuse pneumonia in the patient with AIDS is extensive and often more than one pathogen is present. Clinical features and chest radiographs are rarely diagnostic. Invasive procedures are being replaced by the less invasive diagnostic technique of fibreoptic bronchoscopy and bronchoalveolar lavage. . $^{3-8}$

The increasing incidence of AIDS calls for less expensive and less time consuming methods to obtain a diagnosis. We evaluated a nonbronchoscopic bronchoalveolar lavage technique in intubated patients with AIDS. Our aim was to find a simple, safe, and sensitive technique to diagnose pneumocystis pneumonia and other causes of diffuse pneumonia in these patients.

\section{Methods}

We studied 30 patients with diagnosed or suspected AIDS who developed diffuse pulmonary infiltrates and respiratory failure requiring assisted ventilation. None of the patients required positive end expiratory pressure. The study was approved by the hospital ethics committee and written consent was obtained from the patients.

Tracheal specimens were obtained by standard endotracheal suction. ${ }^{9}$ About 30 minutes before the procedure the inspired oxygen concentration $\left(\mathrm{FIO}_{2}\right)$ was increased to 1.0 ; other ventilator settings were unchanged. Arterial blood gases were measured. A 16 FR $(5 \mathrm{~mm}$ external diameter), $48 \mathrm{~cm}$ Argyle Levin type stomach tube (Sherwood Medical, St Louis, Maryland) was cut proximal to the side ports to leave only a central opening at the distal tip. The tube was inserted into the endotracheal tube via a Portex (Sims Company, Wilming- 
ton, Montana) adaptor and $5 \mathrm{ml}$ of $1 \%$ lignocaine was instilled. The Argyle Levin tube was advanced until resistance was felt. Two $50 \mathrm{ml}$ aliquots of normal saline were instilled and withdrawn by suction via a catheter tipped piston syringe. The Argyle Levin tube was removed and discarded and a repeat arterial blood gas measurement was made 30 minutes later.

Specimens obtained by endotracheal suction and lavage were sent for bacterial, fungal, and cytological studies (including Papanicolaou and silver staining) and direct fluorescent antibody testing for Legionella. A small aliquot of lavage fluid was examined by light microscopy for the presence of alveolar macrophages. The specimen was considered an adequate alveolar sample if numerous alveolar macrophages were seen. ${ }^{10}$

The final diagnosis in cases not diagnosed by our lavage method was made by subsequent fibreoptic bronchoscopy, open lung biopsy, or necropsy or on the basis of the clinical course.

\section{Results}

Tracheal suction specimens were not obtained in 17 of the 30 patients because they had little or no secretion. None of the 15 tracheal specimens sent from the remaining 13 patients was diagnostic.

The mean volume of fluid recovered from lavage was $45 \%$ of that instilled. One specimen was thought to be an inadequate alveolar sample as alveolar macrophages were absent, and this specimen did not provide a diagnosis. Subsequent traditional lavage revealed $P$ carinii. Our lavage technique yielded a diagnosis in 22 of the 30 patients (table). $P$ carinii was found in 21 patients. $P$ carinii and a concomitant infection were found in three patients-two with Mycobacterium aviumintracellulare (cultured from both lavage fluid and blood) and one with cytomegalovirus. One patient had Staphylococcus aureus identified by Gram staining and culture; his clinical picture was consistent with $S$ aureus pneumonia and he responded well to antibiotic treatment. Of the eight for whom lavage failed to yield a diagnosis, three had pneumocystis pneumonia diagnosed by standard bronchoalveolar lavage; endobronchial lesions consistent with Kaposi's sarcoma were seen during fibreoptic broncho-

Diagnoses made by non-bronchoscopic lavage

\begin{tabular}{|c|c|c|c|}
\hline & Diagnosis & $\begin{array}{l}\text { No } \\
\text { diagnosis }\end{array}$ & Total \\
\hline \multicolumn{4}{|l|}{ Pneumonia } \\
\hline Pneumocystis & 21 & 3 & 24 \\
\hline Cytomegalovirus` & 1 & 0 & 1 \\
\hline Staphylococcus aureus & 1 & 0 & 1 \\
\hline Tuberculosis & 0 & 1 & 1 \\
\hline Disseminated MAI* & 2 & 0 & 2 \\
\hline Kaposi's sarcoma & 0 & 1 & 1 \\
\hline Total & 25 & 5 & 30 \\
\hline
\end{tabular}

scopy in another. Mycobacterium tuberculosis was identified in one patient at necropsy. In retrospect one patient was thought to have been in congestive heart failure, and another had resolution of his symptoms and narrowing of his alveolar-arterial oxygen tension difference after correction of his anaemia by blood transfusion. Only one patient remained undiagnosed after fibreoptic bronchoscopy and open lung biopsy.

With our lavage method a specific diagnosis was made on 25 occasions in 30 patients; three patients had more than one cause for their pulmonary disease. A cause for the diffuse pulmonary infiltrates was found therefore in 22 of the 30 patients, the technique thus having a sensitivity of $73 \%(22 / 30)$.

The mean (SD) arterial oxygen tension was $28.7(15.6) \mathrm{kPa}$ before lavage, and $25.0(13.7)$ $\mathrm{kPa}$ after lavage, with a mean decrease of $9.6 \%$; the difference was not significant.

The procedure was completed within 10 minutes in all cases; it was well tolerated, with mild cough in a few of the patients.

\section{Discussion}

Bronchoalveolar lavage is established as a safe, easy, and sensitive procedure for the diagnosis of diffuse pulmonary infiltrates in the immunosuppressed patient. The AIDS epidemic is straining the resources of pulmonary departments in many urban hospitals in the United States. In addition to the many patients needing bronchoscopy, repeated sterilisation reduces the availability and life expectancy of the instrument. A technique requiring less skill that is also less expensive, invasive, and time consuming than fibreoptic bronchoscopy is desirable if it is also safe and sensitive.

In our patients a diagnosis could not be obtained by standard endotracheal suction. Our technique was useful in the diagnosis of pneumocystis, cytomegalovirus, and staphylococcal pneumonia and infection with mycobacteria. The technique was safe, with no significant effect on arterial oxygen tension and no appreciable complications. In the patient who did not have a diagnosis made by this technique who was subsequently diagnosed as having Kaposi's sarcoma the failure to make a diagnosis had little therapeutic implication.

We recommend screening the lavage sample for alveolar macrophages to confirm alveolar sampling. Failure to obtain alveolar samples should be followed by fibreoptic bronchoscopy and traditional bronchoalveolar lavage.

Our lavage method is a simple, safe, and sensitive method for diagnosis. It can be performed quickly, inexpensively, and with little expertise and could be used as the initial diagnostic procedure in this group of patients.

1 Centers for Disease Control. Update: acquired immunodeficiency syndrome-United States. Mortality and Morbidity Weekly Report 1985;34:245-8.

2 Murray JF, Felton CP, Garay SM, et al. Pulmonary complications of the acquired immunodeficiency syndrome: special report of a National Heart, Lung, and drome: special report of a National Heart, Lung, and
Blood Institute workshop. $N$ Engl J Med 1984;310: 1682-8.

3 Golden JA, Hollander H, Stulberg MS, Gamsug G. Bronchoalveolar lavage as the exclusive diagnostic 
modality for Pneumocystis carinii pneumonia. Chest 1986; 90:18-22.

4 Stover DE, Zaman MB, Hajdu SI, Lange $M$, Gold J, Armstrong D. Bronchoalveolar lavage in the diagnosis of diffuse pulmonary infiltrates in the immunosuppresed host. Ann Intern Med 1984;101:1-7.

5 Broaddus C, Dake MD, Stulberg MS, et al. Bronchoalveolar lavage and transbronchial biopsy for the diagnosis of pulmonary infections in the acquired immunodeficiency syndrome. Ann Intern Med 1985;102:747-52.

6 Danielle RP, Elias JA, Epstein PE, Rossman MD. Bronchoalveolar lavage: role in the pathogenesis, diagnosis, and management of interstitial lung disease. Ann Intern Med 1985;102:93-108.
7 Springmeyer SC, Hackman RC, Holis R, et al. Use of bronchoalveolar lavage to diagnose acute diffuse pneumonia in the immunocompromised host. J Infect Dis pneumonia in the

8 Studdy PR, Rudd RM, Gellert AR, Uthayakumar S, Sinha $\mathrm{G}$, Geddes DM. Bronchoalveolar lavage in the diagnosis of diffuse pulmonary shadowing. $\mathrm{Br} J \mathrm{Dis}$ Chest 1984; 78:46-54.

9 DaCunha JP. Sputum specimens. In: Nursing Reference Library. Procedures. Pennsylvania: Springhouse Corporation, 1985:183-5.

10 Lam S, Leriche JC, Kijek K, Phillips D. Effect of bronchial lavage volume on cellular and protein recovery. Chest 1985;88:856-9.

Thorax 1990;45:773-774

\section{Posterior mediastinal}

\section{teratoma with abdominal extension}

\author{
S El Kalla, M Abdul-Hameed, \\ A Al Ashbal, N Menon
}

\begin{abstract}
Posterior mediastinal benign teratomas are uncommon neoplasms in infancy; nine cases have been reported so far. A one month old baby was found to have a benign posterior mediastinal teratoma infiltrating the lower oesophagus from the level of the carina through the oesophageal hiatus into the upper abdomen. Partial oesophagectomy provided a successful outcome.
\end{abstract}

Mediastinal teratomas constitute $11 \cdot 7 \%$ of teratomas in children. ${ }^{1}$ We present a case of benign posterior mediastinal teratoma, rare in

Figure 1 Preoperative barium swallow showing the filling defect at the lower end of the oesophagus and calcification in abdomen and thorax.

\section{Department of} Paediatrics

$S$ El Kalla

N Menon

Department of Cardiothoracic Surgery

M Abdul-Hameed

Department of Pathology

A Al Ashbal

A1 Wasl Maternity and Paediatric Hospital, Dubai, United Arab Emirates

Address for reprint requests: Dr S EI Kalla,

Department of Paediatrics, Department of Health and Medical Services, MOdical Services, United Arab Emirates. United Arab Emirates.

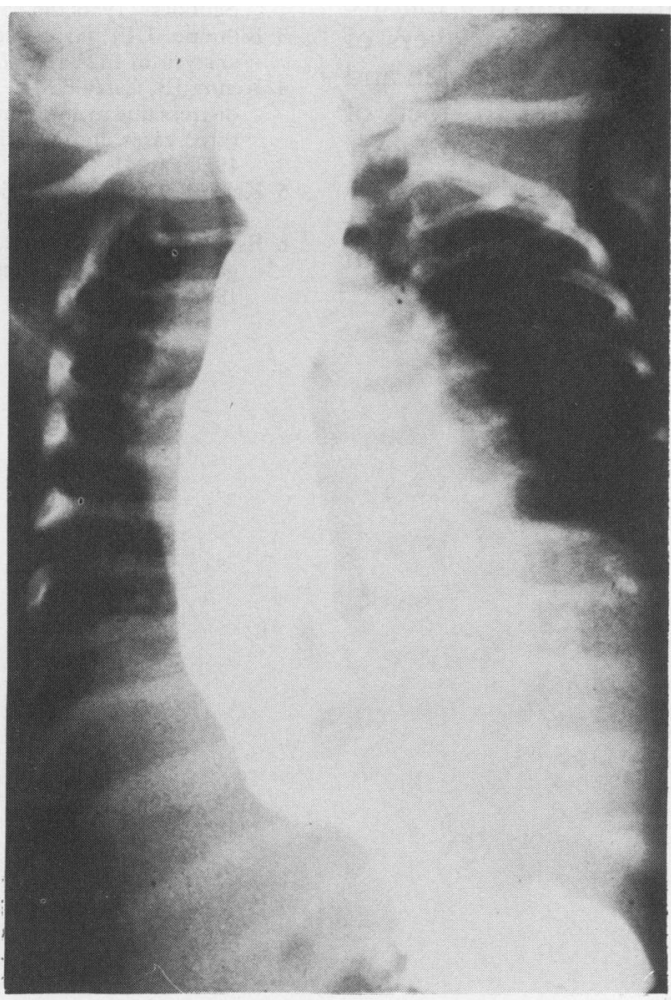

infancy, which was extending into the abdomen.

\section{Case report}

A 1 month old Indian boy presented to $\mathrm{Al}$ Wasl Hospital with a history of cough and breathing difficulty of a week's duration. On examination the infant's indices of growth were found to be normal. He had moderate respiratory distress with decreased breath sounds and dullness to percussion. Abdominal examination disclosed hepatomegaly of 5-6 cm and a palpable left kidney. A firm mass $(5 \times 4 \mathrm{~cm})$ with an ill defined upper margin was later palpated in the epigastrium.

Investigations showed the presence of Escherichia coli in blood and pleural aspirate, which was sensitive to ceftriaxone and gentamicin. Arterial blood gas analysis showed a persistently high carbon dioxide and low oxygen tension. The infant was initially managed with antibiotics, oxygen, and right pleural cavity drainage. A chest radiograph showed right lung consolidation with a pleural effusion and calcification in the posterior mediastinum and abdomen.

The mass was investigated with abdominal ultrasound, intravenous urography, computed tomography of the thorax and abdomen, and a barium swallow with follow through. These showed a large multilobular mass occupying most of the posterior mediastinum and left upper abdomen, extending up to the carina and compressing both lungs and heart. The barium swallow clearly showed that it affected the lower third of the oesophagus, causing mucosal irregularity and leak of barium into the submucosa. The proximal oesophagus was very dilated (fig 1). The mass extended through the oesophageal hiatus into the gastric cardiac fundus, displacing it medially.

A left posterolateral thoracotomy showed a large encapsulated mass in the posterior mediastinum protruding through the oesophageal hiatus. It was intimately adhering to the pericardium, plastering the heart anteriorly to the sternum, and displacing the left lung anterolaterally. It tapered down to end at the carina, to which it seemed to be attached by fibrous tissue. It infiltrated the lower 5-6 cm of the oesophagus. The mass extended through the hiatus into the abdominal cavity for about $4 \mathrm{~cm}$.

Cardio-oesophagectomy was performed. The cardia with a $2 \mathrm{~cm}$ rim of stomach, the affected part of the oesophagus, and a $1 \mathrm{~cm}$ length of normal oesophagus were removed. 